\title{
What is the scale of the future diabetes epidemic, and how certain are we about it?
}

\author{
S. H. Wild • N. G. Forouhi
}

Published online: 21 March 2007

(C) Springer-Verlag 2007

Keywords Diabetes · Diabetes prevalence $\cdot$ Population

\author{
Abbreviations \\ NHANES National Health and Nutrition Education Survey \\ NHIS National Health Interview Survey
}

There is little doubt that worldwide the number of people with diabetes is increasing, and this has led to debate concerning a current or impending epidemic of diabetes $[1,2]$. The reasons for the growth in the number of people with diabetes include population growth and ageing (particularly among ethnic groups that are at a higher risk of diabetes than white European populations, such as South Asians, Afro-Caribbeans, Hispanics and native Americans), the improved survival rate of people with diabetes, changes in diagnostic criteria and screening practices, and alarming increases in the prevalence of overweight and obesity.

The report by Mainous and colleagues in this issue of Diabetologia is the most recent addition to a growing literature on the increasing prevalence of diabetes in the USA [3]. Applying novel refinements to modelling techniques, they estimate that $14.5 \%$ of the entire US adult population (37.7 million people) and $20.2 \%$ of adults of

S. H. Wild

Public Health Sciences, University of Edinburgh,

Edinburgh, UK

N. G. Forouhi $(\bowtie)$

MRC Epidemiology Unit, Elsie Widdowson Laboratory,

Fulbourn Road,

Cambridge CB1 9NL, UK

e-mail: nita.forouhi@mrc-epid.cam.ac.uk
Hispanic origin in the USA will have diabetes by 2031. Do we need yet more projections that shock the world with alarming statistics? And how certain are we about the accuracy of the estimates? In this article we compare these estimates with those derived from other approaches and consider how much confidence can be placed in such figures and their implications.

The likely scale of the problem: projections of diabetes prevalence

The first set of WHO estimates on the global burden of diabetes included data on the projected prevalence in the USA for 2025 [4]. The age- and sex-specific data from these estimates, derived from the combination of reported diabetes prevalence and results of OGTTs obtained during the National Health and Nutrition Education Survey (NHANES) II, performed in 1976-1980, were applied to population projections, giving an estimate of 21.9 million people with diabetes in the USA for 2025 (prevalence $8.9 \%$ ). A similar approach was used in the WHO 2000 Global Burden of Disease study, where data from NHANES III collected between 1988 and 1994 were used to provide an estimate of 30.3 million people with diabetes in the USA by 2030 (prevalence 11.2\%) [5].

The National Health Interview Survey (NHIS) provides data on the prevalence of physician-diagnosed diabetes in the USA obtained during face-to-face interviews. Using NHIS data from 1980-1998, Boyle et al. estimated that there would be approximately 19.9 million people with diagnosed diabetes in the USA in 2025 [6]. Using NHIS data for 1984-2004 and Markov modelling, including data on the increase in diabetes incidence and reduction in the 
relative risk of death among people with diabetes, the same group estimated that 36.4 million people $(10.4 \%)$ will have diagnosed diabetes in the USA in 2030 [7]. Mainous et al. [3] now tell us that if we also take into account the prevalence of individuals at high risk of developing diabetes according to a risk score, then we can expect 37.7 million Americans to have diabetes (diagnosed and undiagnosed but detectable on the basis of an elevated fasting blood glucose) by 2031, with the highest burden among the Hispanic population. Which figures should we believe? And does the variation between them matter?

\section{How certain can we be of the scale of the problem}

Estimates of future prevalence of diabetes are useful for planning, financing and delivery of healthcare. Providing accurate estimates of future prevalence of diabetes is challenging and requires knowledge of the existing prevalence of diabetes in different populations, the relative risks associated with risk factors (including differences in these between population subgroups), accurate estimates of trends in risk factors for diabetes and in population size and characteristics. Errors in these estimates inevitably affect the precision of projections. For example, population estimates and projections are generally based on census data and predicted changes in fertility, mortality and immigration, all of which are subject to error, which is exacerbated by longer projection periods. The nature of the relationship between age and diabetes and the influence of different measures of obesity on risk of diabetes differs between populations [8]. Ideally, estimates of future projections of diabetes prevalence would use appropriate relative risks of risk factors for groups of different age, sex and ethnicity.

The criteria used to identify people with diabetes have a marked effect on prevalence, particularly whether they include both diagnosed and undiagnosed diabetes and which biochemical criteria are used to identify undiagnosed diabetes (especially whether just fasting glucose data or the results of OGTT are used). As the latest estimates from NHANES 1999-2002 suggest that $30 \%$ of diabetes is undiagnosed in the USA [9], then it may be appropriate to adjust the NHIS-based estimates of diagnosed diabetes prevalence so that they can be compared with the estimates, such as those of Mainous et al. [3], based on total (both diagnosed and undiagnosed) diabetes prevalence. Accordingly, if a crude $30 \%$ uplift was applied to latest estimates modelled by the NHIS, the total number of people with diagnosed or undiagnosed diabetes in the USA by 2030 would be expected to be 47.3 million [7]. Even a conservative assumption that $15 \%$ of diabetes was undiagnosed in NHIS participants gives a revised estimate of the total number of people with diagnosed or undiagnosed diabetes in the USA by 2030 of 42.8 million. These are considerably higher than the estimate derived from the approach used by Mainous et al. (37.7 million people with diagnosed or undiagnosed diabetes) [3], highlighting the problems of uncertainty in modelled estimates depending on model inputs and model assumptions.

The estimates provided by Mainous et al. are a useful and welcome addition to the predictions of future prevalence of diabetes as, unlike some previous estimates, they have included data on undiagnosed diabetes, have taken account of changing risk factor patterns, and have included a sensitivity analysis. Their modelling techniques seem methodologically robust, and their use of data from NHANES is appropriate and valid. They acknowledge the limitation of having used a single fasting glucose value to diagnose diabetes, but in the absence of OGTT data, this is a pragmatic approach. The use of a risk score for diabetes to identify high- and low-risk populations for diabetes is an appealing concept. They used the Atherosclerosis Risk in Communities diabetes risk score, which includes the components of the metabolic syndrome (waist circumference, blood pressure, lipids and glucose concentration), with the addition of BMI. It does not include age, sex, family history or ethnicity, but does include sex-specific cut-off points for waist circumference and HDL-cholesterol, and the authors calculated conversion rates for different age/ race groups. A score is assigned from the sum of one point for the presence of each lipid, blood pressure and anthropometric abnormality and two or five points for a fasting glucose value of $>5.6$ or $>6.1 \mathrm{mmol} / \mathrm{l}$, respectively. The high-risk group is defined by a score of more than four points. The sensitivity analysis of varying model assumptions is useful, giving the reader the opportunity to interpret the findings under different assumptions. Interestingly, the authors report that, in terms of the number of people with diabetes, decreases in mortality could be offset by decreases in the proportion of the population who are in the high-risk group. It is in this context that the exact scale of the burden of diabetes becomes less important, and the opportunity for potential prevention of the development of diabetes takes centre stage.

\section{Can we stem the tide?}

Regardless of the exact number of individuals with diabetes, it is clear that the USA (and the rest of the world) will have a substantial burden of diabetes by 2030 , with a greater impact on certain ethnic groups. Several randomised controlled trials have now unequivocally confirmed that lifestyle intervention with an intensive diet and exercise programme is effective in preventing or delaying the onset 
of diabetes among high-risk individuals in diverse ethnic, social and cultural settings such as Finland, the USA, India, Japan and China [10]. A recent meta-analysis estimated a pooled hazard ratio of 0.51 (95\% CI 0.44 0.60 ) for lifestyle interventions vs standard advice [11]. Several trials have also confirmed that oral hypoglycaemic drugs are effective in the primary prevention of diabetes among high-risk individuals, with a pooled hazard ratio of 0.70 (95\% CI 0.62-0.79) for oral diabetes drugs vs control [11]. However, there is ongoing debate as to whether glucose-lowering can really be labelled as prevention [10]. Lifestyle changes offer a long-lasting effect on diabetes risk reduction, well beyond the duration of the trial [12], and lifestyle goals have major potential to prevent diabetes at the population level [13]. These are powerful arguments for investing in the primary prevention of diabetes by lifestyle management.

In his American Diabetes Association presidential address in 2005, Alan Cherrington recommended a fourpronged approach to tackling the increasing burden of diabetes: to invest in research, to adopt a chronic care model for the treatment of diabetes, to focus on the development of affordable and effective prevention strategies, and to control and prevent obesity [14]. Given the calculation by Mainous et al. that a one percentage point drop in the US population estimate of diabetes in adults ( $>20$ years old) in 2031 is equivalent to a decrease in the prevalence of diabetes of 2.6 million people, there is a powerful incentive to implement these approaches.

\section{Conclusion}

Increasingly sophisticated models to estimate the burden of diabetes have emerged in the last few years, with the latest model described by Mainous et al. [3] incorporating demographic factors, incidence, mortality and migration, as well as estimates of trends in the proportion of the population at high risk of diabetes. All estimates of the scale of the burden of diabetes in the USA and the rest of the world suggest a major future health burden, with the exact size of the burden being difficult to ascertain with confidence.

While the importance of obtaining a reasonable estimate of the total prevalence of diabetes in a country for the planning and delivery of health services is acknowledged, it is not an adequate end in itself. Research efforts must focus on addressing the wide-ranging challenges that these estimates raise: what can be done to delay or prevent the complications of diabetes (secondary and tertiary prevention), and what can be done to decrease the future projected burden, i.e. delay or prevent the onset of diabetes itself (primary prevention)? Increasing knowledge about the future burden of diabetes must precipitate action. Urgent implementation and further development of evidence-based health, education, transport, and food policy to tackle the obesogenic environment is now required [15-17]. We must all rise to this challenge.

\section{References}

1. Zimmet P, Lefebvre P (1996) The global NIDDM epidemic. Treating the disease and ignoring the symptom. Diabetologia 39:1247-1248

2. Wareham NJ, Forouhi NG (2005) Is there really an epidemic of diabetes? Diabetologia 48:1454-1455

3. Mainous AG 3rd, Baker R, Koopman RJ et al (2007) Impact of the population at risk of diabetes on projections of diabetes burden in the United States: an epidemic on the way. Diabetologia, DOI 10.1007/s00125-006-0528-5

4. King H, Aubert RE, Herman WH (1998) Global burden of diabetes, 1995-2025: prevalence, numerical estimates, and projections. Diabetes Care 21:1414-1431

5. Wild S, Roglic G, Green A et al (2004) Global prevalence of diabetes: estimates for the year 2000 and projections for 2030 . Diabetes Care 27:1047-1053

6. Boyle JP, Honeycutt AA, Narayan KM et al (2001) Projection of diabetes burden through 2050: impact of changing demography and disease prevalence in the U.S. Diabetes Care 24:1936-1940

7. Narayan KM, Boyle JP, Geiss LS et al (2006) Impact of recent increase in incidence on future diabetes burden: U.S., 2005-2050. Diabetes Care 29:2114-2116

8. Glumer C, Vistisen D, Borch-Johnsen K et al (2006) Risk scores for type 2 diabetes can be applied in some populations but not all. Diabetes Care 29:410-414

9. Cowie CC, Rust KF, Byrd-Holt DD et al (2006) Prevalence of diabetes and impaired fasting glucose in adults in the U.S. population: National Health And Nutrition Examination Survey 1999-2002. Diabetes Care 29:1263-1268

10. Tuomilehto J (2007) Counterpoint: evidence-based prevention of type 2 diabetes: the power of lifestyle management. Diabetes Care $30: 435-438$

11. Gillies CL, Abrams KR, Lambert PC et al (2007) Pharmacological and lifestyle interventions to prevent or delay type 2 diabetes in people with impaired glucose tolerance: systematic review and meta-analysis. BMJ 334:299

12. Lindstrom J, Ilanne-Parikka P, Peltonen M et al (2006) Sustained reduction in the incidence of type 2 diabetes by lifestyle intervention: follow-up of the Finnish Diabetes Prevention Study. Lancet 368:1673-1679

13. Simmons RK, Harding AH, Jakes RW et al (2006) How much might achievement of diabetes prevention behaviour goals reduce the incidence of diabetes if implemented at the population level? Diabetologia 49:905-911

14. Cherrington AD (2006) 2005 Presidential address: diabetes: past, present, and future. Diabetes Care 29:2158-2164

15. Gostin LO (2007) Law as a tool to facilitate healthier lifestyles and prevent obesity. JAMA 297:87-90

16. Lean M, Gruer L, Alberti G et al (2006) ABC of obesity. Obesitycan we turn the tide? BMJ 333:1261-1264

17. World Health Organization Regional Office for Europe (2006) European Charter on counteracting obesity. http://www.euro.who. int/Document/E89567.pdf, last accessed in February 2007 